\title{
II. LEG 55 SEDIMENT GRAIN SIZE AND CARBON/CARBONATE DATA
}

\author{
The Leg 55 Scientific Staff
}

\section{CARBONATE BOMB}

$\mathrm{CaCO}_{3}$ content was determined on board ship by the "Karbonat Bomb" technique (Müller and Gastner, 1971). In this simple procedure, a sample is powdered and treated with $\mathrm{HCl}$ in a closed cylinder. Any resulting $\mathrm{CO}_{2}$ pressure is proportional to the $\mathrm{CaCO}_{3}$ content of the sample. Application of the calibration factor to the manometer reading $(\times 100)$ yields per cent $\mathrm{CaCO}_{3}$. The error can be as low as 1 per cent for sediments high in $\mathrm{CaCO}_{3}$, and in general an accuracy of \pm 2 to 5 per cent can be obtained.

These data are presented on the Core Description Forms for each site.

\section{CARBON-CARBONATE ANALYSIS}

After the cruise, sediment samples were analyzed at the DSDP sediment laboratory on a LECO WR-12 Carbon Analyzer. Sample preparation procedures are identical to those used with the old LECO 70 Analyzer, as outlined in Boyce and Bode (1972) and Bode (1973). Discussions of the LECO WR-12 Analyzer are presented in Bode (1979). Accuracy and precision of the results are as follows:

$$
\begin{array}{ll}
\text { Total carbon } & = \pm 0.3 \% \text { (absolute) } \\
\text { Organic carbon } & = \pm 0.06 \% \text { (absolute) } \\
\mathrm{CaCO}_{3} & = \pm 3 \% \text { (absolute) }
\end{array}
$$

The carbon-carbonate data are presented in Table 1.

\begin{tabular}{|c|c|c|c|}
\hline $\begin{array}{l}\text { Core-Section, } \\
\text { Interval }(\mathrm{cm})\end{array}$ & $\begin{array}{l}\text { Total } \\
\text { Carbon }\end{array}$ & $\begin{array}{l}\text { Organic } \\
\text { Carbon }\end{array}$ & $\mathrm{CaCO}_{3}$ \\
\hline \multicolumn{4}{|l|}{ Hole 430A } \\
\hline $\begin{array}{l}1-1,41 \\
2-1,125 \\
4-1,10 \\
4-1,34\end{array}$ & $\begin{array}{r}9.9 \\
8.9 \\
10.7 \\
0.2\end{array}$ & $\begin{array}{l}0.1 \\
1.0 \\
0.1 \\
0.1\end{array}$ & $\begin{array}{r}82 \\
66 \\
88 \\
1\end{array}$ \\
\hline \multicolumn{4}{|l|}{ Hole 431A } \\
\hline $\begin{array}{l}1-1,45 \\
2-1,69\end{array}$ & $\begin{array}{l}3.3 \\
0.2\end{array}$ & $\begin{array}{l}0.1 \\
0.0\end{array}$ & $\begin{array}{r}26 \\
2\end{array}$ \\
\hline \multicolumn{4}{|l|}{ Hole 432} \\
\hline $1-4,104$ & 6.7 & 0.2 & 55 \\
\hline \multicolumn{4}{|l|}{ Hole 432A } \\
\hline $2, \mathrm{CC}, 25$ & 0.1 & 0.1 & 0 \\
\hline \multicolumn{4}{|l|}{ Hole 433} \\
\hline $\begin{array}{l}1-1,98 \\
1-3,55\end{array}$ & $\begin{array}{l}3.5 \\
8.6\end{array}$ & $\begin{array}{l}0.1 \\
0.2\end{array}$ & $\begin{array}{l}28 \\
70\end{array}$ \\
\hline
\end{tabular}

TABLE 1

Carbon and Carbonate Analyses

\begin{tabular}{lccc}
\multicolumn{4}{c}{ TABLE 1-Continued } \\
\hline $\begin{array}{c}\text { Core-Section, } \\
\text { Interval (cm) }\end{array}$ & $\begin{array}{c}\text { Total } \\
\text { Carbon }\end{array}$ & $\begin{array}{c}\text { Organic } \\
\text { Carbon }\end{array}$ & $\mathrm{CaCO}_{3}$ \\
\hline Hole 433A & & & \\
2-1, 145 & 7.5 & 0.2 & 61 \\
$3-1,140$ & 0.9 & 0.1 & 6 \\
$3-2,91$ & 9.9 & 0.3 & 80 \\
$3-5,44$ & 10.3 & 0.1 & 85 \\
$4-1,55$ & 6.8 & 0.3 & 54 \\
$4-2,107$ & 3.9 & 0.4 & 29 \\
$4-6,40$ & 7.3 & 0.2 & 59 \\
$10-3,53$ & 11.5 & 0.1 & 95 \\
$12-1,95$ & 10.7 & 0.1 & 89 \\
$14-1,65$ & 11.1 & 0.1 & 91 \\
$16-1,62$ & 11.3 & 0.1 & 93 \\
$19-1,19$ & 10.5 & 0.1 & 87 \\
& & & \\
Hole 433B & & & \\
2-1, 32 & 11.1 & 0.1 & 91 \\
$2-1,107$ & 11.7 & 0.1 & 97 \\
$3-1,44$ & 11.1 & 0.1 & 92 \\
\hline
\end{tabular}

\section{GRAIN SIZE ANALYSIS}

Distribution of sand-size, silt-size, and clay-size particles was determined from $10-\mathrm{cm}^{3}$ sediment samples at the DSDP sediment laboratory by standard sieve and pipette methods (Appendix III of Volume IV, Initial Reports of the Deep Sea Drilling Project, p. 745, with modified settling times as in Boyce, 1972). Grain size data are listed in Table 2. Textures in Table 2 use Shepard's (1954) sediment classification. The sand, silt, and clay boundaries are based on the Wentworth (1922) scale. Thus, the particle sizes of the sand, silt, and clay fractions range from 2000 to $62.5 \mu \mathrm{m}, 62.5$ to $3.91 \mu \mathrm{m}$, and less than $3.91 \mu \mathrm{m}$, respectively.

\section{REFERENCES}

Bode, G. W., 1979. Grain-size and carbon/carbonate analyses, Leg 45. In Melson, W. G., Rabinowitz, P. O., et al., Initial Reports of the Deep Sea Drilling Project, v. 45: Washington (U. S. Government Printing Office), pp. 379380.

1973. Carbon and carbonate analyses-Leg 18. In Kulm, L. D., von Huene, R., et al., Initial Reports of the Deep Sea Drilling Project, v. 18: Washington (U. S. Government Printing Office), pp. 1069-1076.

Boyce, R. E., 1972. Grain size analysis, Leg 9. In Hays, H. D., et al., Initial Reports of the Deep Sea Drilling Project, v. 9: Washington (U. S. Government Printing Office), pp. 779796.

Boyce, R. E. and Bode, G. W., 1972. Carbon and carbonate analyses, Leg 9. In Hays, J. D., et al., Initial Reports of the 
TABLE 2

Grain Size Analyses

\begin{tabular}{|c|c|c|c|c|}
\hline $\begin{array}{l}\text { Core-Section, } \\
\text { Interval }(\mathrm{cm})\end{array}$ & $\%$ Sand & $\%$ Silt & $\%$ Clay & Classification \\
\hline \multicolumn{5}{|l|}{ Hole 430} \\
\hline $1-4,48$ & 74.4 & 13.5 & 12.0 & Silty sand \\
\hline \multicolumn{5}{|l|}{ Hole 430A } \\
\hline $1-1,8$ & 66.9 & 18.1 & 15.0 & Silty sand \\
\hline $2-1,40$ & 73.4 & 17.8 & 8.8 & Silty sand \\
\hline \multicolumn{5}{|l|}{ Hole 431A } \\
\hline $2-1,72$ & 16.8 & 50.1 & 73.1 & Clayey silt \\
\hline \multicolumn{5}{|l|}{ Hole 432} \\
\hline $1-2,77$ & 36.7 & 50.2 & 13.0 & Sandy silt \\
\hline $1-4,82$ & 89.3 & 5.8 & 5.0 & Sand \\
\hline \multicolumn{5}{|l|}{ Hole 433} \\
\hline $1-1,123$ & 31.8 & 47.1 & 21.1 & Sand-Silt-Clay \\
\hline $1-3,48$ & 3.5 & 34.9 & 61.6 & Silty clay \\
\hline \multicolumn{5}{|l|}{ Hole 433A } \\
\hline $2-1,140$ & 3.1 & 44.5 & 52.4 & Silty clay \\
\hline $3-2,70$ & 1.5 & 31.6 & 66.9 & Silty clay \\
\hline $3-5,45$ & 0.7 & 26.1 & 73.2 & Silty clay \\
\hline $4-1,50$ & 5.9 & 32.8 & 61.3 & Silty clay \\
\hline $4-2,58$ & 24.6 & 38.7 & 36.7 & Sand-Silt-Clay \\
\hline $4-3,60$ & 8.4 & 35.7 & 55.9 & Silty clay \\
\hline $4-6,68$ & 3.7 & 33.6 & 62.8 & Silty clay \\
\hline $5-2,138$ & 1.7 & 30.3 & 68.1 & Silty clay \\
\hline $5-4,32$ & 1.9 & 22.8 & 75.3 & Clay \\
\hline $5-5,45$ & 2.0 & 29.6 & 68.4 & Silty clay \\
\hline $6-1,60$ & 2.8 & 29.5 & 67.7 & Silty clay \\
\hline $6-2,92$ & 1.3 & 27.9 & 70.8 & Silty clay \\
\hline $6-3,100$ & 3.3 & 26.2 & 70.5 & Silty clay \\
\hline $6-4,70$ & 1.8 & 28.8 & 69.4 & Silty clay \\
\hline $6-5,80$ & 2.9 & 29.4 & 67.6 & Silty clay \\
\hline $6-6,20$ & 1.2 & 26.5 & 72.3 & Silty clay \\
\hline $6-6,95$ & 4.3 & 35.3 & 60.4 & Silty clay \\
\hline
\end{tabular}

TABLE 2 - Continued

\begin{tabular}{cccrl}
\hline $\begin{array}{c}\text { Core-Section, } \\
\text { Interval }(\mathrm{cm})\end{array}$ & \% Sand & \% Silt & \% Clay & Classification \\
\hline $6-6,127$ & 1.1 & 34.0 & 65.0 & Silty clay \\
6-7, 3 & 0.4 & 42.2 & 57.4 & Silty clay \\
$6-7,37$ & 24.1 & 42.9 & 33.0 & Sand-Silt-Clay \\
$7-1,70$ & 72.3 & 12.3 & 15.3 & Clayey sand \\
$7-5,80$ & 66.6 & 12.8 & 20.6 & Clayey sand \\
$7-6,91$ & 58.9 & 19.4 & 21.7 & Clayey sand \\
$8-1,36$ & 91.6 & 2.9 & 5.5 & Sand \\
$8-1,146$ & 63.8 & 14.3 & 21.9 & Clayey sand \\
$8-3,11$ & 31.9 & 22.4 & 45.6 & Sand-Silt-Clay \\
$8-5,69$ & 62.5 & 17.5 & 20.0 & Clayey sand \\
$8-7,30$ & 34.7 & 34.9 & 30.4 & Sand-Silt-Clay \\
$9-2,36$ & 77.8 & 7.2 & 15.0 & Sand \\
$9-6,8$ & 93.8 & 1.3 & 4.8 & Sand \\
$10-6,94$ & 37.0 & 33.8 & 29.2 & Sand-Silt-Clay \\
$12-1,92$ & 69.8 & 23.2 & 7.0 & Silty sand \\
$14-1,66$ & 70.8 & 11.2 & 18.0 & Clayey sand \\
$16-1,63$ & 68.6 & 13.0 & 18.4 & Clayey sand \\
$19-1,28$ & 93.0 & 1.9 & 5.1 & Sand \\
Hole 433B & & & & \\
$1-1,34$ & 75.5 & 10.1 & 14.5 & Sand \\
$1-4,129$ & 61.7 & 15.7 & 22.6 & Clayey sand \\
Hole 433C & & & & \\
3-2,109 & 95.9 & 0.5 & 3.6 & Sand \\
\hline
\end{tabular}

Deep Sea Drilling Project, v. 9: Washington (U. S. Government Printing Office), pp. 797-816.

Müller, G. and Gastner, M., 1971. The "Karbonat-Bomb", a simple device for the determination of the carbonate content in sediments, soils and other materials, $N$. Jb. Miner, Mh., v. 10, pp. 466-469.

Shepard, F. P., 1954. Nomenclature based on sand-silt-clay ratios, J. Sediment. Petrol., v. 24, pp. 151-158.

Wentworth, C. K., 1922. A scale of grade and class terms of clastic sediments, J. Geol., v. 30, pp. 377. 